\title{
SoLAT (Sorafenib Lenvatinib alternating treatment): a new treatment protocol with alternating Sorafenib and Lenvatinib for refractory thyroid Cancer
}

Soo Young Kim ${ }^{1,2,3+}$, Seok-Mo Kim ${ }^{1,2,3+}$, Ho-Jin Chang ${ }^{1,2,3}$, Bup-Woo Kim ${ }^{1,2,3}$, Yong Sang Lee ${ }^{1,2,3}$, Cheong Soo Park ${ }^{1,2,3}$, Ki Cheong Park ${ }^{2^{*}+}$ and Hang-Seok Chang ${ }^{1,2,3^{*}+}$

\begin{abstract}
Background: In the last decade, several tyrosine kinase inhibitors (TKIs), which disrupt pathways involved in the proliferation and tumorigenesis of thyroid cancer, have been extensively studied. Two different TKIs, lenvatinib and sorafenib, were recently approved by both the US FDA and European Medicine Agency. Until date, the duration of the TKI response is not sufficient and resistance eventually occurs. The goal of this study was to investigate a new treatment protocol, SoLAT, using sorafenib and lenvatinib alternatively on refractory thyroid cancer.

Methods: Patient-derived aggressive papillary thyroid cancer (PTC) cell lines from patients with biochemical and histologically proven aggressive RAI-refractory papillary thyroid cancer were exposed to sorafenib and lenvatinib alternatively. Human thyroid cancer cell xenografts were obtained by injecting patient-derived aggressive PTC cell lines into the flank of female BALB/c nude mice. Tumor-bearing mice were treated with sorafenib and lenvatinib alternatively. Cell viability assay, immunofluorescence analysis, confocal imaging, immunoblot analysis, flow cytometry analysis of cell cycle and a tube formation assay were performed.

Results: SoLAT was more effective for advanced PTC cell lines than individual treatment. Immunoblot analysis showed that SoLAT markedly increased levels of cell cycle inhibitors (p53 and p21), and pro-apoptotic factors (Apaf-1 and cleaved caspase 3) and decreased levels of positive cell cycle regulators (cyclin D1, CDK4, CDK6) and anti-apoptotic factors ( $p-N F K B, B C l-2$ ). Increased sub- $G_{0} / G_{1}$ population was observed in the SoLAT group, leading to apoptosis, cell cycle arrest, and strong inhibition of advanced PTC cell viability. SoLAT reduced the level of EMT markers such as vimentin, E-cadherin, Snail and Zeb1 by FGFR inhibition. In the xenograft model, individual treatment with sorafenib or lenvatinib did not markedly suppress patient-derived aggressive PTC cell xenograft tumors, whereas SoLAT significantly suppressed the proliferation of these tumors.

Conclusions: SoLAT was more effective than individual treatment with sorafenib or lenvatinib in inhibiting PTC progression by inducing cell cycle arrest. Studies using both in vitro cell culture and an in vivo xenograft model provided evidence of tumor shrinkage with SoLAT. We suggest that these effects may be due to reduced EMT-mediated drug resistance in the aggressive PTC model.
\end{abstract}

Keywords: Papillary thyroid cancer, Lenvatinib, Sorafenib, EMT, FGF signaling, TKI

\footnotetext{
* Correspondence: ggiru95@yuhs.ac; SURGHSC@yuhs.ac

†Soo Young Kim, Seok-Mo Kim, Ki Cheong Park and Hang-Seok Chang contributed equally to this work.

${ }^{2}$ Department of Surgery, Yonsei University College of Medicine, 50-1,

Yonsei-ro, Seodaemun-gu, Seoul 120-752, South Korea

1 Thyroid Cancer Center, Gangnam Severance Hospital, Department of

Surgery, Yonsei University College of Medicine, Seoul 120-720, South Korea

Full list of author information is available at the end of the article
}

(c) The Author(s). 2018 Open Access This article is distributed under the terms of the Creative Commons Attribution 4.0 International License (http://creativecommons.org/licenses/by/4.0/), which permits unrestricted use, distribution, and reproduction in any medium, provided you give appropriate credit to the original author(s) and the source, provide a link to the Creative Commons license, and indicate if changes were made. The Creative Commons Public Domain Dedication waiver (http://creativecommons.org/publicdomain/zero/1.0/) applies to the data made available in this article, unless otherwise stated. 


\section{Background}

Thyroid cancer accounts for more than 90\% of all endocrine cancers and is the most common endocrine malignancy, as its incidence has increased over the last three decades [1]. Thyroid cancer is distinguished into well-differentiated, poorly differentiated, and anaplastic thyroid cancer based on cell differentiation characteristics and the ability to maintain follicular cell features. Differentiated thyroid cancer (DTC) is the most common thyroid cancer, representing more than $90 \%$ of all thyroid carcinomas. DTC is characterized by papillary and follicular histological subtypes [2, 3]. However, advanced cancer subtypes, including anaplastic thyroid cancer (ATC), have poor prognosis due to resistance to treatment and aggressive behavior [4], with total median survival of only few months [5]. Recently, novel targeted therapies have increased the lifespan of cancer patients. Kinase inhibitors are recommended for treating radioactive iodine (RAI)-refractory differentiated thyroid cancer (DTC) patients with metastatic, rapidly progressive, symptomatic, and/or imminently threatening disease that is not otherwise amenable to local control using alternative approaches [6]. Nevertheless, this has not been the case for patients with advanced cancer subtypes. Recent studies have revealed molecules and mechanisms that are closely connected to poor clinical outcomes in advanced thyroid cancer [7, 8]. Among these mechanisms, we concentrated on the epithelial-mesenchymal transition (EMT) and EMT-induced drug resistance of cancer stem cells (CSCs) as one of the probable reasons for the poor clinical results [9, 10]. EMT of cancer cells not only induces metastasis, but also contributes to drug resistance [10-12]. Therefore, it is necessary to determine the specific molecular changes or mechanisms of thyroid carcinogenesis to overcome the depressing outcome associated with advanced thyroid cancer.

Sorafenib was the first tyrosine kinase inhibitor tested in a phase III trial and was approved for the treatment of metastatic DTC in 2013. Patients with progressive RAI-refractory DTC treated with oral sorafenib showed improved progression-free survival compared to patients receiving placebo [13]. Lenvatinib was tested in RAI-refractory DTC patients in a phase III trial and was approved for use in RAI-resistant metastatic DTC in $2015[14,15]$. The most important difference between lenvatinib and other drugs is its ability to inhibit fibroblast growth factor receptor 1 (FGFR1), making it an effective drug for cases with resistance to vascular endothelial growth factor/receptor (VEGF/R) inhibitors [16]. In reality, patients who were treated successively with sorafenib and lenvatinib or vice versa did not have any further treatment option. In metastatic renal cell cancer, the sequential use of two tyrosine kinase inhibitors was tested, showing that there was no difference in progression-free survival dependent on the sequence of the two drugs $[17,18]$. A protocol of sequential alternating treatment regimen of tyrosine kinase inhibitor and chemotherapy was studied in non-small cell lung cancer [19]. Since there are no other treatment options than sorafenib and levatinib led to the hypothesis that alternating the use of sorafenib or lenvatinib may be a better and more effective way of treating refractory thyroid cancer than using one single agent alone.

In this study, we delineated the mechanism of drug resistance of cancer cells by inhibiting FGFR signaling and EMT in response to current treatments and discussed how these problems are being addressed.

\section{Methods}

\section{Patients/tissue specimens}

Fresh tumors were gained from patients with biochemical and histologically proven aggressive RAI-refractory papillary thyroid cancer who were treated at the Thyroid Cancer Center, Gangnam Severance Hospital, Yonsei University College of Medicine, Seoul, Korea. Further protocol and details are described in our previous articles [20,21].

\section{Tumor cell isolation and primary culture}

After resection, the tumors were transported to the laboratory. Normal tissue and fat were eliminated and rinsed with $1 \times$ Hank's balanced salt solution (HBSS). Additional protocol and details are indicated in our previous article [21]. The research protocol was approved by the Institutional Review Board of the Thyroid Cancer Center, Gangnam Severance Hospital, Yonsei University College of Medicine (IRB Protocol: 3-2016-0076).

\section{Cell culture}

The patient-derived aggressive papillary thyroid cancer (PTC) cell lines were grown in RPMI-1640 medium with 10 15\% FBS. Authentication of the cell lines were carried out by with Cell ID system (Promega, Corporation, Madison, WI, USA) comparing their profiles with those published on the DMSZ database. Mycoplasma contamination was invariably checked with the Lookout Mycoplasma PCR Detection Kit (Sigma-Aldrich; MP0035).

\section{Cell viability assay}

Cell proliferation was measured using the 3-(4, 5-dimethylthiazol-2-yl)-2, 5-diphenyl tetrazolium bromide (MTT) assay. Additional protocol is described in our previous article [20].

\section{SoLAT (Sorafenib Lenvatinib alternating treatment) In vitro}

At first, the combination treatment of the Sorafenib and Lenvatinib for 5 days, after Sorafenib and Lenvatinib alternating treatment for 5 days. 


\section{In vivo}

At first, the combination treatment of the Sorafenib and Lenvatinib for 10 days, after Sorafenib and Lenvatinib alternating treatment for 10 days.

\section{Immunofluorescence analysis and confocal imaging}

$\beta$-catenin expression was analyzed with immunofluorescent staining. Further protocol and data analysis details are described in our previous article [20].

\section{Immunoblot analysis}

Antibodies against Ki-67 (Abcam), cyclin D1 (Santa Cruz Biotechnology, Dallas, TX, USA), CDK4 (Santa Cruz Biotechnology), p21 (Santa Cruz Biotechnology), p53 (Santa Cruz Biotechnology), p-ERK 1/2 (Santa Cruz Biotechnology), ERK 1/2 (Santa Cruz Biotechnology), Apaf-1 (Abcam), p-NFkB (Santa Cruz Biotechnology), Bcl-2 (Santa Cruz Biotechnology), caspase 3 (Santa Cruz Biotechnology), vimentin (Abcam), E-cadherin (Abcam), Snail (Abcam), Zeb1 (Abcam), and $\beta$-actin (Santa Cruz Biotechnology) overnight at $4{ }^{\circ} \mathrm{C}$.

\section{Flow cytometry analysis of the cell cycle}

Cells were treated with sorafenib and lenvatinib alone in an alternating regimen (SoLAT) in RPMI-1640 medium containing $10 \%$ FBS for $40 \mathrm{~h}$, harvested by trypsinization, and fixed with $70 \%$ ethanol. Further protocol and data analysis details are described in our previous articles [20, 21].

\section{Tube formation assay}

Human umbilical vein endothelial cells (HUVECs) $(7 \times$ $10^{4}$ ) were cultured with growth factor-reduced Matrigel (BD Biosciences, San Jose, CA, USA) in MV1 for $1 \mathrm{~h}$ for cell attachment, following which the endothelial growth basal medium-2 (EBM-2) was replaced with conditioned medium and cell culture was continued for $24 \mathrm{~h}$. Tube length was quantified after $8 \mathrm{~h}$ by measuring the total cumulative tube length in 3 random microscopic fields with a computer-assisted microscope using Image J software. The original magnification used was $\times 100$.

\section{Human thyroid cancer cell xenografts}

The patient-derived aggressive PTC cells $\left(3.5 \times 10^{6}\right.$ cells/ mouse) were cultured in vitro and then injected subcutaneously into the upper left flank region of female BALB/c nude mice. After 11 days, tumor-bearing mice were grouped randomly ( $n=10 /$ group) and $10 \mathrm{mg} / \mathrm{kg}$ lenvatinib (administered p.o.) and $40 \mathrm{mg} / \mathrm{kg}$ sorafenib (administered p.o.), or lenvatinib or sorafenib alone (administered p.o.) were administered once every two days. Tumor size was measured on alternative days using calipers.. All experiments were approved by the Animal Experiment Committee of the Yonsei University.

\section{Immunohistochemistry}

Primary Antibodies against p21 (Santa Cruz Biotechnology) and Bcl-2 (Abcam) were diluted with PBS at a ratio of 1:100 and incubated overnight at $4{ }^{\circ} \mathrm{C}$. All tissue sections were counterstained with hematoxylin, dehydrated, and mounted.

\section{Statistical analysis}

Statistical analyses were performed using GraphPad Prism software (GraphPad Software Inc., La Jolla, CA, USA). Immunohistochemistry results were subjected to ANOVA followed by a Bonferroni post hoc test. Values are expressed as the mean \pm SD. $P<0.05$ indicated statistical significance.

\section{Results}

Sorafenib and lenvatinib TKIs do not completely inhibit the proliferation of patient-derived PTC cells

To investigate the anti-cancer effects of the TKIs sorafenib and lenvatinib on patient-derived PTC cells, we assayed GSP2 and GSP3 (Fig. 1a) cell proliferation in the presence of these compounds by the MTT assay (Fig. 1b). Concentration-dependent inhibition was not complete, although it was sufficient to determine the $\mathrm{IC}_{50}$ of sorafenib and lenvatinib in GSP2 and GSP3 cells (Fig. 1c).

\section{SoLAT is more effective than individual treatment in tumor suppression}

We investigated the anti-cancer activity of alternating treatment of sorafenib and lenvatinib (SoLAT) on advanced PTC. Individual treatments with sorafenib or lenvatinib did not significantly inhibit advanced PTC. SoLAT was more effective than the individual treatments (Fig. 2a and d). We performed immunofluorescence (Fig. $2 \mathrm{~b}$ and e) and immunoblot analyses of cell cycle markers such as Ki-67 to confirm this observation (Fig. 2c and f). Sorafenib or lenvatinib treatment alone did not show any significant difference in Ki-67 levels compared to the control group. However, SoLAT suppressed Ki-67 expression (Fig. 2b and e). Evaluation of cell cycle-related protein levels by immunoblot analysis yielded similar results (Fig. 2c and $\mathrm{f}$ ). A marked increase in the levels of p53, p21 (well-known inhibitors of the cell cycle), Apaf-1, and cleaved caspase 3 (pro-apoptotic factors) and decrease in the levels of cyclin D1, CDK 4, CDK 6 (positive regulators of the cell cycle), $\mathrm{p}-\mathrm{NF} \mathrm{K}$, and $\mathrm{Bcl}-2$ (anti-apoptotic factors) were observed compared to sorafenib or lenvatinib treatment alone. These results conclusively show that cancer cell proliferation was inhibited by SoLAT. 


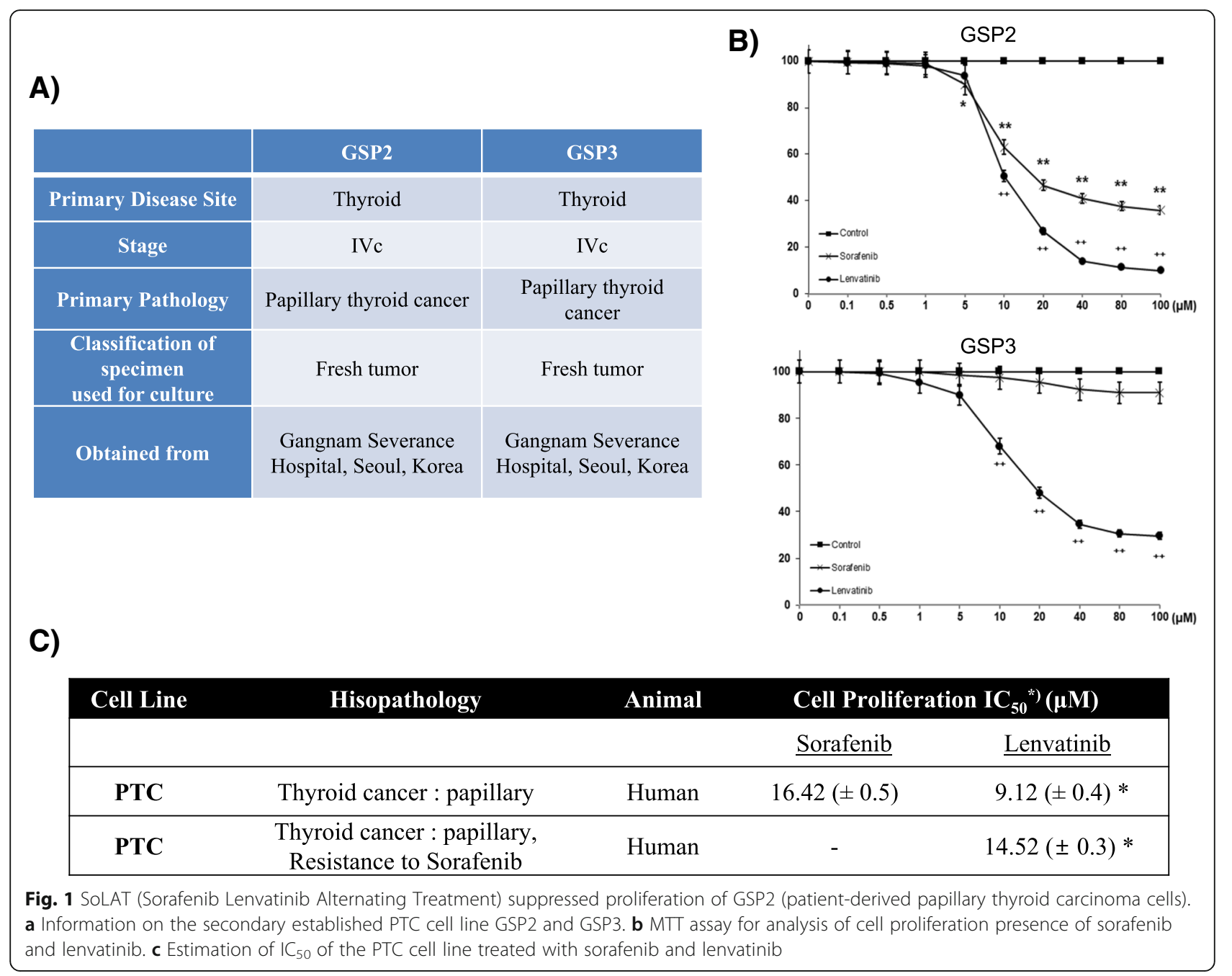

SoLAT is more efficient than the individual treatments in inducing cell cycle arrest, although it also increases drug resistance in advanced PTC

SoLAT increased the sub- $G_{0} / G_{1}$ population $(P<0.05)$ and induced cell death in advanced PTC, GSP2 and GSP3 (Table 1). Thus, the synergistic effect of sorafenib and lenvatinib potently induced the sub- $G_{0} / G_{1}$ population, leading to apoptosis, cell cycle arrest, and strong inhibition of advanced PTC viability. However, a small increase in the sub- $G_{0} / G_{1}$ population was observed after SoLAT, indicating that drug resistance was also induced by this treatment in advanced PTC.

\section{SoLAT reduces EMT-mediated drug resistance in advanced PTC}

Lenvatinib is well-known for reducing drug resistance-associated EMT by inhibiting FGFR. However, the patient-derived advanced PTC cells used in this study showed high levels of drug resistance. Consequently, no significant inhibition of drug resistance was achieved by individual sorafenib and lenvatinib treatments. However, SoLAT reduced the level of certain EMT markers. Immunofluorescence assay confirmed that SoLAT inhibited nuclear translocation of $\beta$-catenin in advanced PTC cells more potently than either agent alone (Fig. $3 a$ and c). In addition, the levels of most EMT markers (vimentin, E-cadherin, Snail, and Zeb1) were reduced by FGFR inhibition (p-ERK 1/2) in the SoLAT group (Fig. $3 b$ and d). This demonstrates that SoLAT effectively decreased EMT-mediated drug resistance via FGFR inhibition in advanced PTC.

Angiogenesis of aggressive PTC is suppressed by SoLAT VEGF secretion activates angiogenesis, and SoLAT reduced angiogenic activity by suppressing VEGF secretion compared to the no treatment or individual treatment groups (Fig. 4a and b). Next, we used the VEGF-induced tube formation assay with HUVECs to analyze the effect 


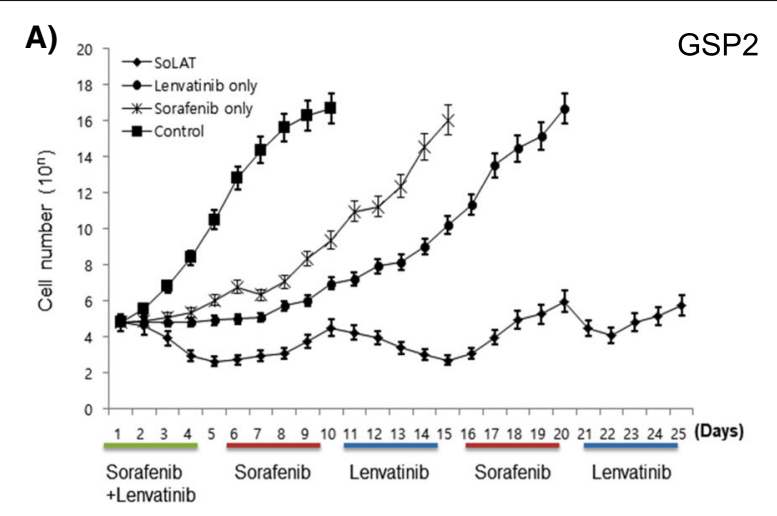

C)

B)
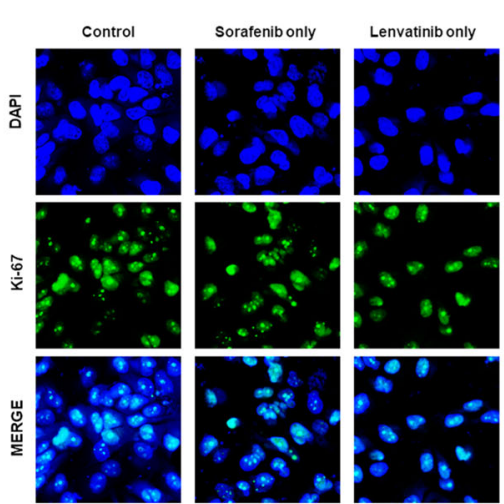

D)

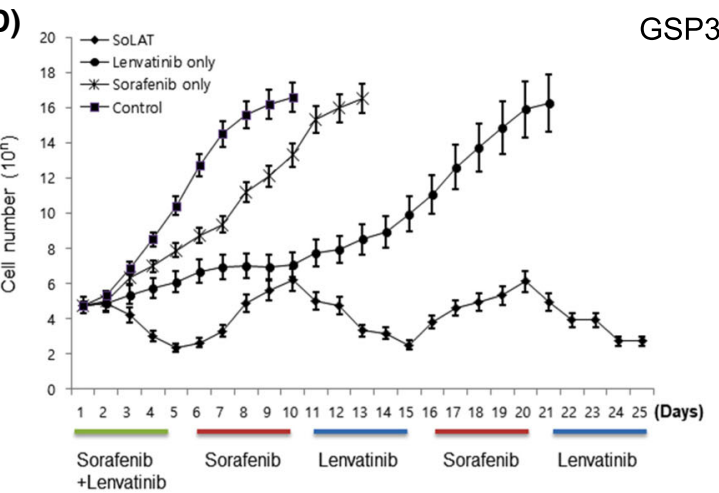

E)
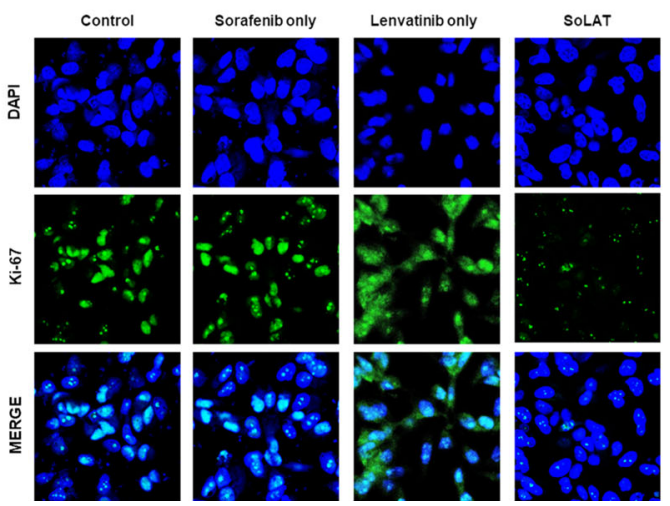

SOLAT
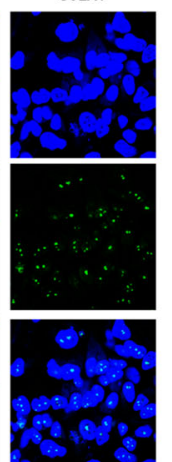

GSP3
F)

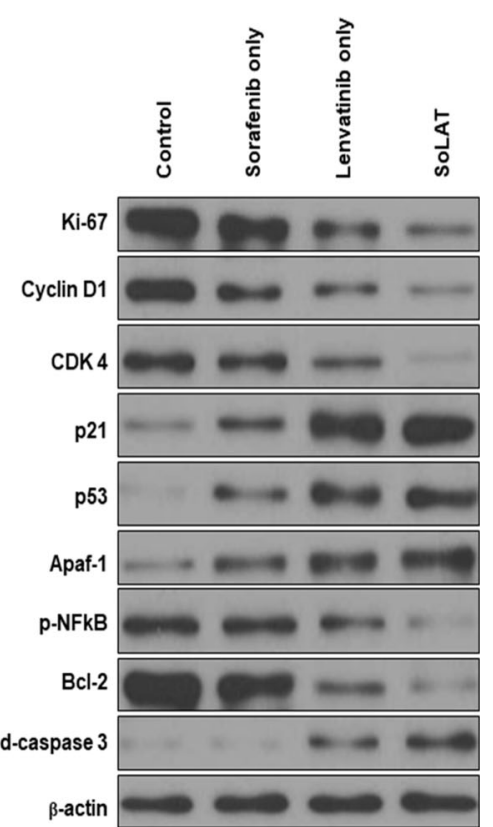

Fig. 2 (See legend on next page.) 
(See figure on previous page.)

Fig. 2 SoLAT was more efficiently induced cell cycle arrest and reduced anti-apoptotic factor than each treated groups on GSP2 and GSP3. a and $\mathbf{d}$ Anti-cancer activity of SoLAT on the advanced PTC cell line. Sorafenib Lenvatinib alternating treatment SoLAT was more efficient than the individual treatments. $\mathbf{b}$ and $\mathbf{e}$ Immunofluorescence staining for Ki-67. The SoLAT group suppressed Ki-67 expression. $\mathbf{c}$ and $\mathbf{f}$ Immunoblot analysis of cell-cycle arrest and apoptotic proteins showed marked increase in the levels of p21, p53, Apaf-1, and cleaved caspase 3, and decrease in the levels of Ki-67, cyclin D1, CDK 4, p-NFkB, and BCl-2 in the SoLAT group than in the individual sorafenib or lenvatinib treatment groups

of switching treatment on angiogenesis. HUVECs were cultured in the conditioned media of advanced PTC cells. After $16 \mathrm{~h}$ of culture, we evaluated the formation of a tubular structure with sorafenib or lenvatinib treatment alone and with SoLAT. The tube length in the advanced PTC-conditioned media alone was higher than that in the drug treatment groups (Fig. 4b). Quantitation of the tube length revealed that SoLAT in PTC-conditioned media considerably decreased the tube length compared to the no-treatment group and individual treatments (Fig. 4b). This demonstrated that secreted VEGF was inhibited by SoLAT.

SoLAT induces tumor shrinkage in the xenograft model Individual treatments with sorafenib or lenvatinib did not markedly suppress patient-derived aggressive PTC cell xenograft tumors; however, SoLAT significantly suppressed the proliferation of these tumors (Fig. 5a and d). No evidence of systemic toxicity or treatment-related death was observed in any group. Sorafenib or lenvatinib treatment did not significantly affect the body weight of mice (Fig. 5b and e). The SoLAT group showed significantly smaller tumor volumes than the individual sorafenib or lenvatinib treatment groups (Fig. 5c and f). Anti-apoptotic activity is a crucial factor for assessing the biological behavior of tumors. The levels of Bcl-2 and p21, which are common anti-apoptosis and cell cycle arrest markers, respectively, were determined by immunohistochemical and immunoblot analysis examination of patient-derived aggressive PTC cell xenograft tumors. SoLAT maximally decreased Bcl-2 and increased p21 levels (Fig. 6a, b and c). Thus, all of the results conclusively show that SoLAT exerts potent anti-cancer activity in the aggressive PTC cell xenograft model.

\section{Discussion}

To our knowledge, this is the first study which showed that alternating treatment with the TKIs lenvatinib and sorafenib (SoLAT) was more effective than individual

Table 1 Cell cycle analysis: Alternating treatment with sorafenib and lenvatinib SoLAT showed significant increase in the sub- $G_{0} / G_{1}$ population and induction of cell death in advanced PTC (GSP2 and GSP3)

\begin{tabular}{|c|c|c|c|c|c|}
\hline \multicolumn{2}{|l|}{ Status } & Sub- $\mathrm{G}_{0} \mathrm{G}_{1}$ & $\mathrm{G}_{0} \mathrm{G}_{1}$ & S & $\mathrm{G}_{2} / \mathrm{M}$ \\
\hline \multicolumn{6}{|l|}{ GSP2 } \\
\hline \multicolumn{2}{|l|}{ Control } & $1.4 \pm 0.05$ & $49.5 \pm 0.05$ & $27.2 \pm 0.09$ & $21.9 \pm 0.05$ \\
\hline \multicolumn{2}{|c|}{ Sorafenib only } & $4.7 \pm 0.08$ & $51.3 \pm 0.07$ & $25.4 \pm 0.09$ & $18.6 \pm 0.05$ \\
\hline \multicolumn{2}{|c|}{ Lenvatinib only } & $9.8 \pm 0.04$ & $49.6 \pm 0.03$ & $27.4 \pm 0.12$ & $13.2 \pm 0.04$ \\
\hline \multirow[t]{5}{*}{ SoLAT } & $S+L$ & $29.8 \pm 0.09$ & $46.7 \pm 0.15$ & $18.9 \pm 0.05$ & $4.6 \pm 0.03$ \\
\hline & Sorafenib & $7.5 \pm 0.02$ & $49.5 \pm 0.07$ & $30.6 \pm 0.01$ & $12.4 \pm 0.08$ \\
\hline & Lenvatinib & $21.9 \pm 0.04$ & $50.1 \pm 0.12$ & $22.3 \pm 0.05$ & $5.7 \pm 0.02$ \\
\hline & Sorafenib & $1.7 \pm 0.07$ & $49.3 \pm 0.04$ & $28.5 \pm 0.05$ & $20.5 \pm 0.09$ \\
\hline & Lenvatinib & $13.7 \pm 0.15$ & $48.3 \pm 0.02$ & $27.5 \pm 0.01$ & $10.5 \pm 0.02$ \\
\hline \multicolumn{6}{|l|}{ GSP3 } \\
\hline \multicolumn{2}{|l|}{ Control } & $1.1 \pm 0.03$ & $49.8 \pm 0.08$ & $27.5 \pm 0.15$ & $21.6 \pm 0.12$ \\
\hline \multicolumn{2}{|c|}{ Sorafenib only } & $2.4 \pm 0.04$ & $51.6 \pm 0.06$ & $26.4 \pm 0.05$ & $19.6 \pm 0.01$ \\
\hline \multicolumn{2}{|c|}{ Lenvatinib only } & $13.6 \pm 0.04$ & $53.2 \pm 0.03$ & $24.5 \pm 0.07$ & $8.7 \pm 0.05$ \\
\hline \multirow[t]{5}{*}{ SOLAT } & $S+L$ & $36.2 \pm 0.15$ & $45.6 \pm 0.14$ & $14.3 \pm 0.19$ & $3.9 \pm 0.22$ \\
\hline & Sorafenib & $2.7 \pm 0.15$ & $51.2 \pm 0.12$ & $26.7 \pm 0.12$ & $19.4 \pm 17$ \\
\hline & Lenvatinib & $32.4 \pm 0.11$ & $49.5 \pm 0.21$ & $13.5 \pm 0.07$ & $4.6 \pm 0.15$ \\
\hline & Sorafenib & $2.4 \pm 0.15$ & $52.8 \pm 0.25$ & $27.6 \pm 0.14$ & $17.2 \pm 0.13$ \\
\hline & Lenvatinib & $22.9 \pm 0.24$ & $55.1 \pm 0.13$ & $15.5 \pm 0.19$ & $6.5 \pm 0.15$ \\
\hline
\end{tabular}




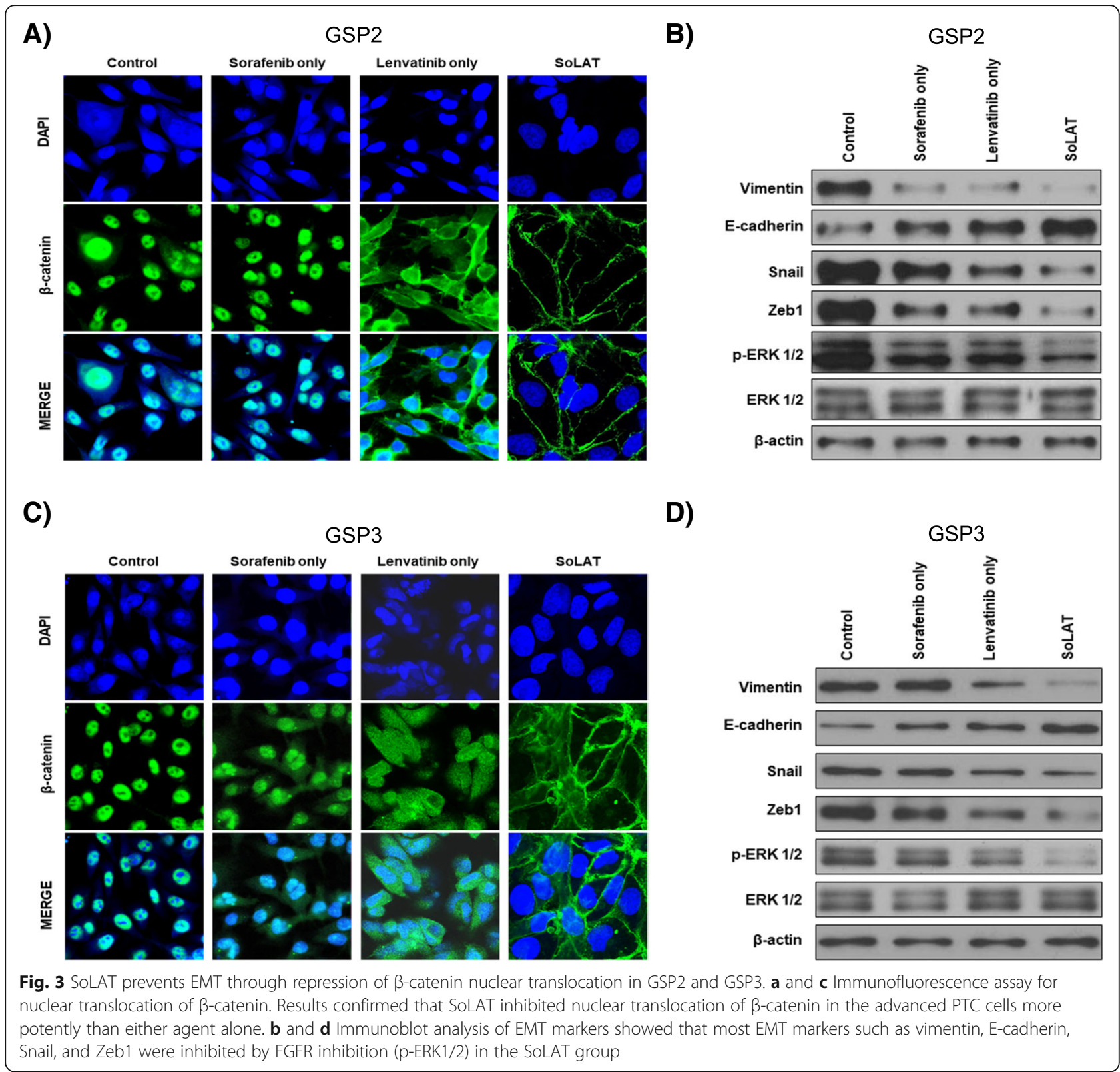

treatment with sorafenib or lenvatinib in inhibiting PTC tumor progression by inducing cell cycle arrest. There have been studies testing alternating chemoradiotherapy instead of concurrent chemoradiation for nasopharyngeal cancer and studies which showed alternating treatment for mantle cell lymphoma [22, 23]. Sequential use of two tyrosine kinase inhibitors was tested in metastatic renal cell carcinoma [17].

Studies using both in vitro cell culture and in vivo xenograft model provided evidence of tumor shrinkage in the alternative switching group. We suggest that these effects may be due to reduced EMT-mediated drug resistance in the aggressive PTC model.
EMT is induced in aggressive forms of thyroid cancer with elevated ZEB1 levels, which can promote drug resistance through EMT-dependent and EMT-independent mechanisms [24-26]. Studies have shown that downregulation of $Z E B 1$ could restore drug sensitivity [27, 28]. Sorafenib inhibits EMT in hepatocellular carcinoma, attenuates HGF secretion in polarized macrophages, decreases plasma HGF levels, and abolishes polarized macrophage-induced activation of the HGF receptor Met [29]. EMT reversal was shown to overcome drug resistance in lung adenocarcinoma [30].

The frequency and nature of adverse side effects differ between sorafenib and lenvatinib. Hand foot skin 


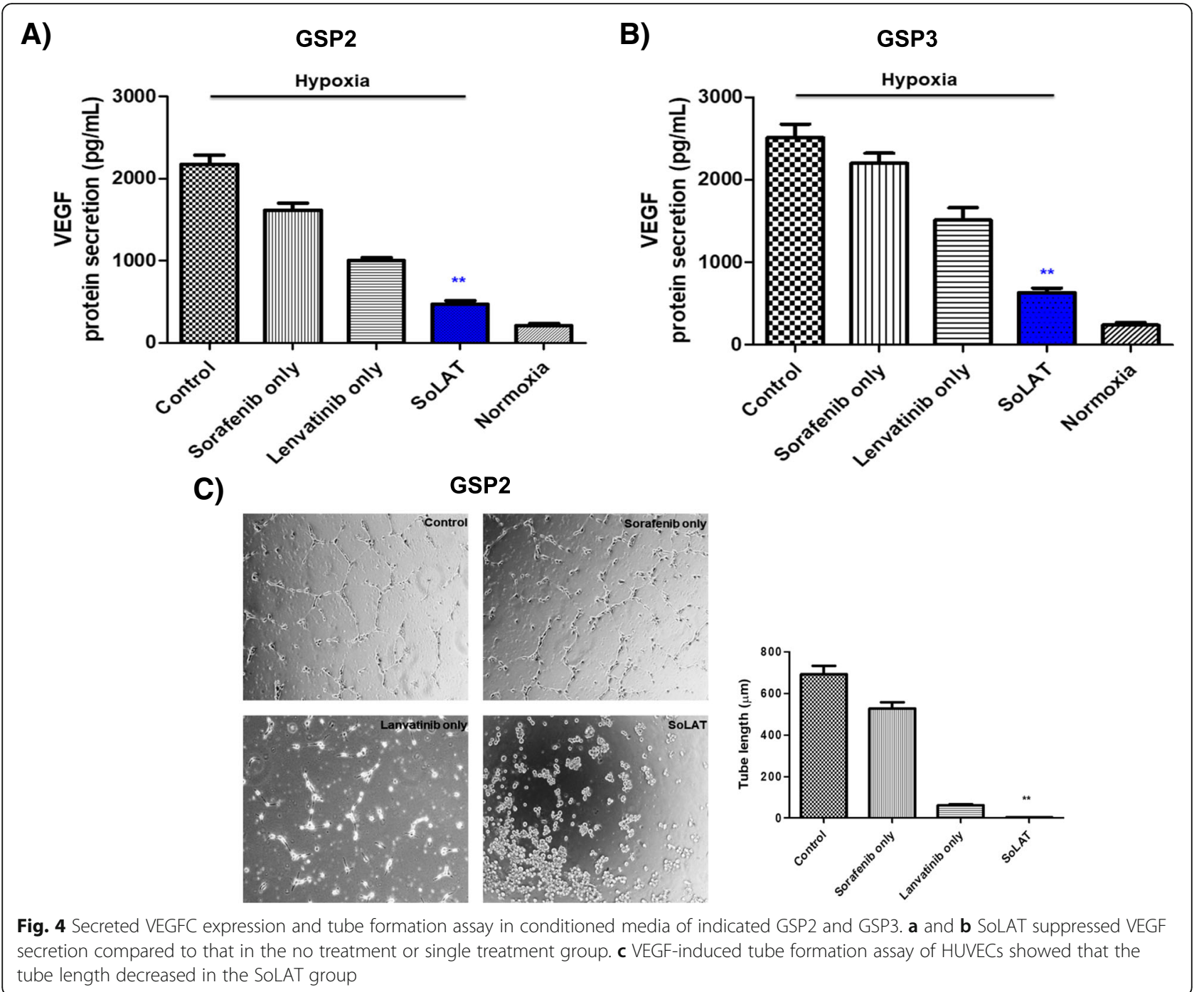

reaction is the most common side effect of sorafenib, whereas hypertension is the most common adverse effect of lenvatinib. Our results show that the $\mathrm{IC}_{50}$ is reduced when the drugs are used alternately compared to when used individually, which suggests that this can be an option for decreasing drug toxicity. In contrast to combination therapy, the drugs may be effective before being washed out of the body in the case of interval treatment; however, this requires further in vivo evidence. These findings may assist in developing a treatment protocol with reduced toxicity and enhanced drug efficacy.

TKIs are recommended for the treatment of RAI-refractory DTC patients with metastatic, rapidly progressive, symptomatic, and/or imminently threatening disease, which is not otherwise amenable to local control using alternative approaches. The benefits of systemic therapeutics have been demonstrated in the form of improved progression-free survival in three randomized, double-blinded, placebo-controlled clinical trials for vandetanib, sorafenib, and lenvatinib $[6,14,15]$. Sorafenib is known to inhibit RAF-1, a member of the RAF/MEK/ERK signaling pathway, and also BRAF, VEGFR-2, VEGFR-3, PDGFR- $\beta$, and c-KIT [31]. Lenvatinib has a potent inhibitory effect on VEGFR-2, VEGFR-3, PDGFR $\alpha / \beta$, KIT, RET, and FGFR1-4. Lenvatinib differs from other drugs in its ability to inhibit FGFR1, providing efficacy in cases with VEGFR inhibitor resistance [16, 32, 33]. Despite favorable results in phase III trials and their status as the first line of treatment for RAI-refractory DTCs, both lenvatinib and sorafenib eventually elicit toxicity, and most patients discontinue them owing to unresponsiveness. A second-line kinase inhibitor therapy such as lenvatinib should be considered for patients with disease progression during initial kinase inhibitor therapy without prohibitive adverse effects [6]. 

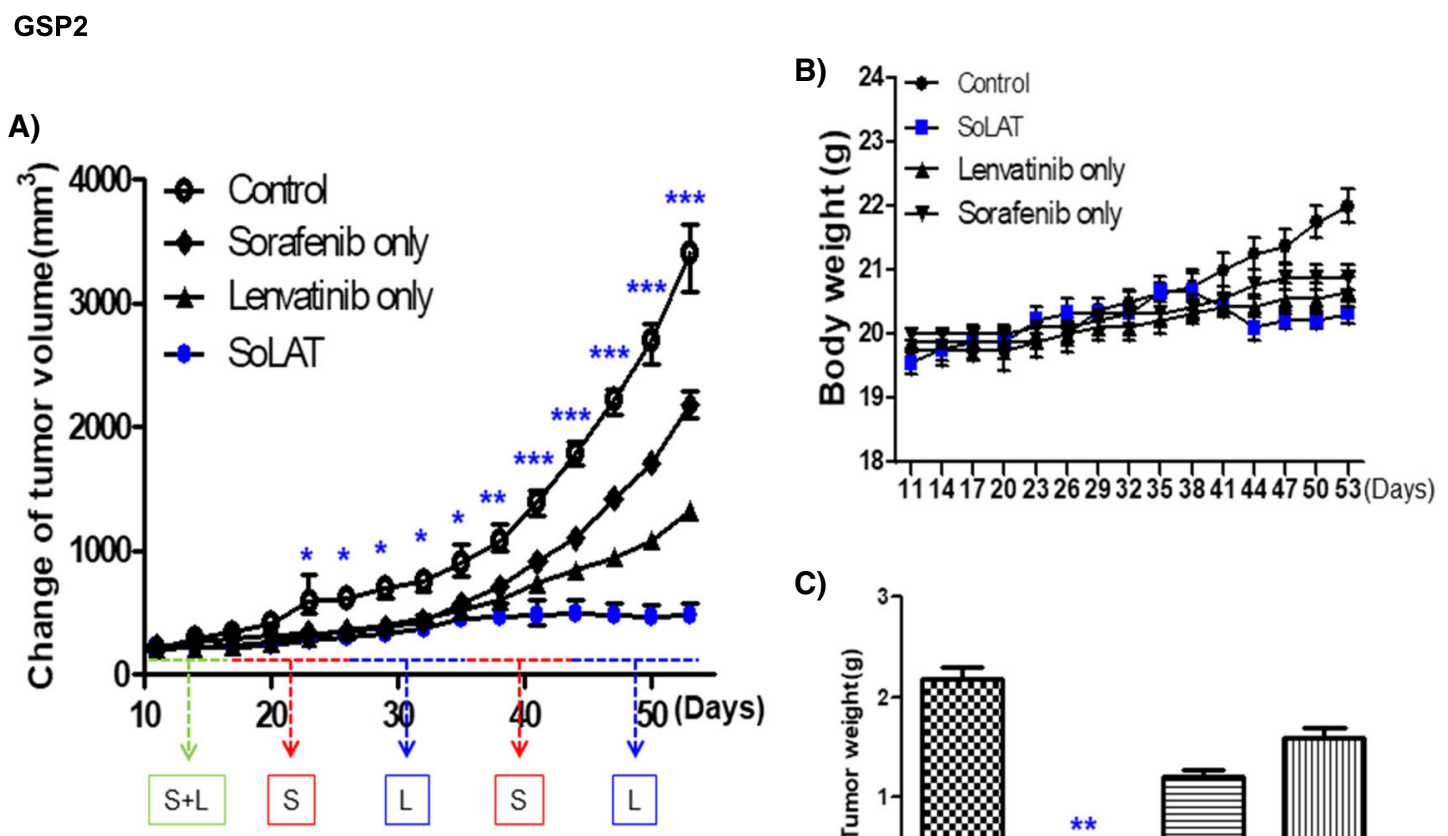

C)
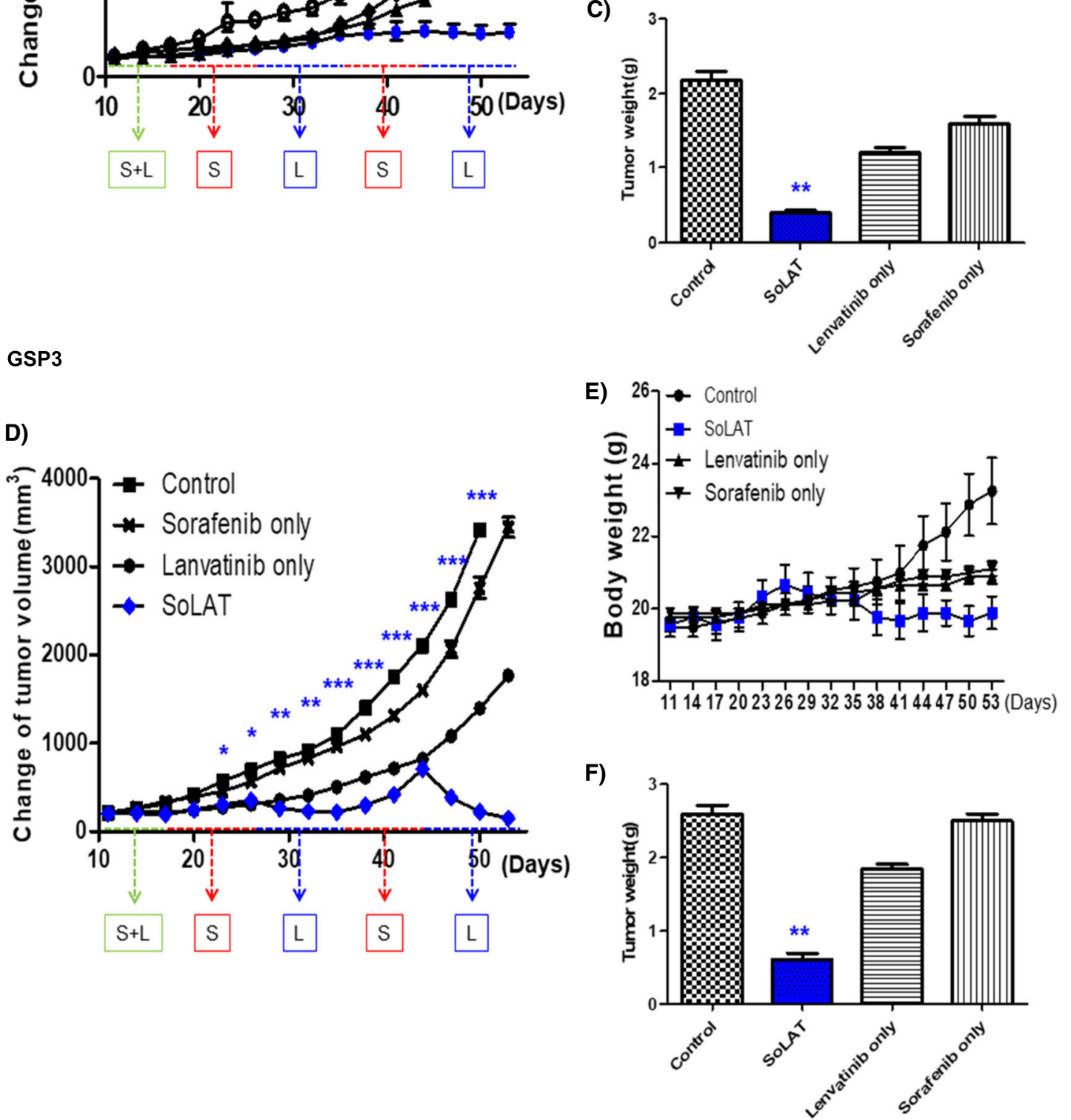

Fig. 5 (See legend on next page.) 
(See figure on previous page.)

Fig. 5 SoLAT was most efficiently induced tumor shrinkage in GSP2 and GSP3 xenografts model. a and $\mathbf{d}$ SoLAT suppressed tumor growth better than individual treatments with sorafenib or lenvatinib. $\mathbf{b}$ and $\mathbf{e}$ Sorafenib or lenvatinib treatment did not significantly affect the body weight of treated mice. No evidence of systemic toxicity or treatment-related death was observed in any group. $\mathbf{c}$ and $\mathbf{f}$ The SoLAT group showed significantly smaller tumor volumes compared to the individual treatment groups

Mechanisms for TKI resistance include receptor autophosphorylation, autophagy, involvement of hypoxia-inducing factor, epigenetic regulation, and EMT [34, 35]. Furthermore, several EMT-inducing cytokines such as TGF- $\beta$, FGF, HGF, insulin-like growth factor, and IL-6 may also be involved $[30,36]$. We noted that SoLAT blocked constitutive ERK phosphorylation. The RAS/ RAF/MEK/ERK signaling pathway is a major signaling pathway for EMT and metastasis, and inhibition of this pathway significantly reduces EMT [37]. Because we only analyzed ERK as a marker of this pathway in this study, other markers should be investigated in the future to confirm the FGFR inhibition-mediated EMT-reducing effects of lenvatinib.
It is necessary to investigate the mechanism underlying the success of the alternating treatment with lenvatinib and sorafenib compared to that of individual lenvatinib treatment in inhibiting the growth of aggressive PTC both in vitro and in vivo. Further studies are required to compare the efficiencies and toxicities of combination therapy and SoLAT. In addition, the effect of reusing one drug after development of resistance to both drugs (as an alternating therapy or combination therapy) has to be determined. Similarly, the effectiveness of reusing a therapeutic agent after development of resistance versus alternative interval treatment for prolonging disease-free survival has to be evaluated; in addition, the appropriate

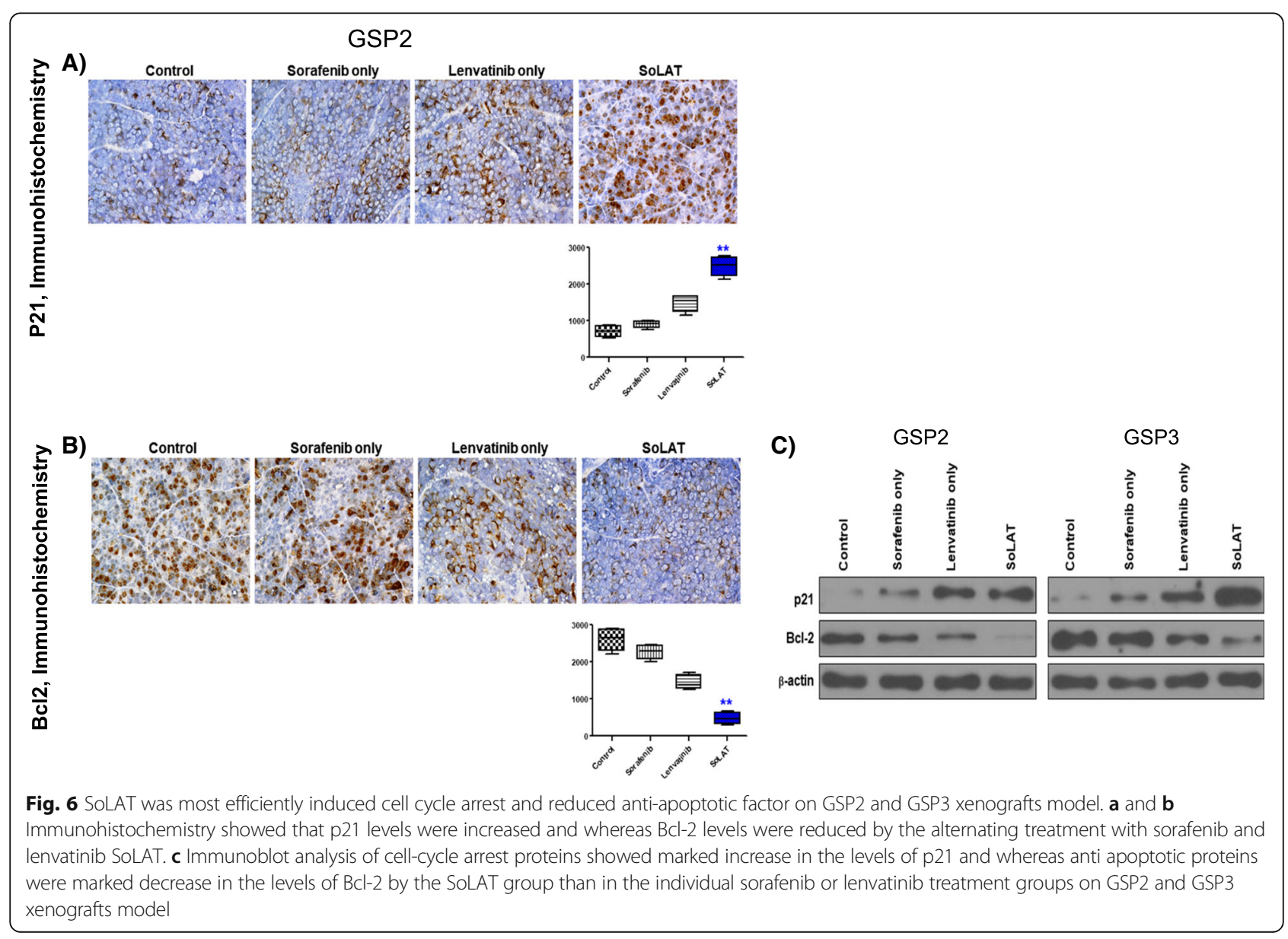


interval should be determined if interval treatment is found to be more efficient.

\section{Conclusions}

The current study suggests that SoLAT was more effective than individual treatment with sorafenib or lenvatinib in inhibiting PTC progression by inducing cell cycle arrest and reducing EMT-mediated drug resistance.

\section{Abbreviations}

EMT: epithelial mesenchymal transition; FGF: fibroblast growth factor; PTC: Papillary thyroid cancer, lenvatinib, sorafenib; TKI: tyrosine kinase inhibitor

\section{Acknowledgments}

The authors thank Dr. Hyeung-Kyoo Kim for critical brainstorming and assistance with the clinical research.

\section{Funding}

Our working group has not received any financial support. This study was only financially supported by a faculty research grant of Yonsei University College of Medicine (6-2016-0038)

\section{Availability of data and materials}

The datasets used and/or analysed during the current study are available from the corresponding author on reasonable request.

\section{Consent to publish}

Also included korean-speaking parents or guardians of child respondents for inter-rater reliability assessment.

\section{Authors' contributions}

SYK and SMK mainly carried out the in vitro and in vivo studies. HJC and BWK were involved in drafting the manuscript. SYK contributed to the statistical analysis. SYK, YSL, and CSP were also involved in drafting the manuscript and study design. KCP and HSC mainly designed the study, developed the concept, performed the experiments, wrote the manuscript, and approved the final version for submission.

\section{Ethics approval and consent to participate}

The study will be performed according to the Declaration of Helsinki. All animal experiments were approved by the Animal Experiment Committee of Yonsei University, Korea (2017-0276). GSP2 and GSP3 are donations required human ethics approval. Ethical approval was acquired by the Institutional Review Board of the Thyroid Cancer Center, Gangnam Severance Hospital, Yonsei University College of Medicine (IRB Protocol: 3-2016-0076).

\section{Competing interests}

The authors declare that they have no competing interests.

\section{Publisher's Note}

Springer Nature remains neutral with regard to jurisdictional claims in published maps and institutional affiliations.

\section{Author details}

${ }^{1}$ Thyroid Cancer Center, Gangnam Severance Hospital, Department of Surgery, Yonsei University College of Medicine, Seoul 120-720, South Korea. ${ }^{2}$ Department of Surgery, Yonsei University College of Medicine, 50-1, Yonsei-ro, Seodaemun-gu, Seoul 120-752, South Korea. ${ }^{3}$ Yonsei Institute of Refractory Thyroid Endocrine Cancer, Yonsei University, Seoul, South Korea.

Received: 24 March 2018 Accepted: 24 September 2018 Published online: 04 October 2018

\section{References}

1. Nikiforov YE. Thyroid carcinoma: molecular pathways and therapeutic targets. Mod Pathol. 2008;21(Suppl 2):S37-43.
2. Schlumberger MJ. Papillary and follicular thyroid carcinoma. N Engl J Med. 1998:338(5):297-306.

3. Soares P, Lima J, Preto A, Castro P, Vinagre J, Celestino R, Couto JP, Prazeres H, Eloy C, Maximo V, et al. Genetic alterations in poorly differentiated and undifferentiated thyroid carcinomas. Curr Genomics. 2011;12(8):609-17.

4. Smallridge RC. Approach to the patient with anaplastic thyroid carcinoma. J Clin Endocrinol Metab. 2012;97(8):2566-72

5. Gullu BE, Celik O, Gazioglu N, Kadioglu P. Thyroid cancer is the most common cancer associated with acromegaly. Pituitary. 2010;13(3):242-8.

6. Haugen BR, Alexander EK, Bible KC, Doherty GM, Mandel SJ, Nikiforov YE, Pacini F, Randolph GW, Sawka AM, Schlumberger M, et al. 2015 American Thyroid Association management guidelines for adult patients with thyroid nodules and differentiated thyroid Cancer: the American Thyroid Association guidelines task force on thyroid nodules and differentiated thyroid Cancer. Thyroid. 2016;26(1):1-133.

7. Bernet V, Smallridge R. New therapeutic options for advanced forms of thyroid cancer. Expert Opin Emerg Drugs. 2014;19(2):225-41.

8. Ma R, Minsky N, Morshed SA, Davies TF. Stemness in human thyroid cancers and derived cell lines: the role of asymmetrically dividing cancer stem cells resistant to chemotherapy. J Clin Endocrinol Metab. 2014;99(3):E400-9.

9. Mani SA, Guo W, Liao MJ, Eaton EN, Ayyanan A, Zhou AY, Brooks M, Reinhard F, Zhang CC, Shipitsin M, et al. The epithelial-mesenchymal transition generates cells with properties of stem cells. Cell. 2008;133(4):704-15.

10. Grygielewicz P, Dymek B, Bujak A, Gunerka P, Stanczak A, LamparskaPrzybysz M, Wieczorek M, Dzwonek K, Zdzalik D. Epithelial-mesenchymal transition confers resistance to selective FGFR inhibitors in SNU-16 gastric cancer cells. Gastric Cancer. 2016;19(1):53-62.

11. Housman G, Byler S, Heerboth S, Lapinska K, Longacre M, Snyder N, Sarkar S. Drug resistance in cancer: an overview. Cancers (Basel). 2014;6(3):1769-92.

12. Kerbel RS. Molecular and physiologic mechanisms of drug resistance in cancer: an overview. Cancer Metastasis Rev. 2001;20(1-2):1-2.

13. Dang RP, McFarland D, Le VH, Camille N, Miles BA, Teng MS, Genden EM, Misiukiewicz KJ. Neoadjuvant therapy in differentiated thyroid Cancer. Int $J$ Surg Oncol. 2016;2016:3743420.

14. Schlumberger M, Tahara M, Wirth LJ, Robinson B, Brose MS, Elisei R, Habra MA, Newbold K, Shah MH, Hoff AO, et al. Lenvatinib versus placebo in radioiodine-refractory thyroid cancer. N Engl J Med. 2015;372(7):621-30.

15. Brose MS, Nutting CM, Jarzab B, Elisei $R$, Siena $S$, Bastholt $L$, de la Fouchardiere C, Pacini F, Paschke $R$, Shong YK, et al. Sorafenib in radioactive iodinerefractory, locally advanced or metastatic differentiated thyroid cancer: a randomised, double-blind, phase 3 trial. Lancet. 2014;384(9940):319-28.

16. Lorusso L, Pieruzzi L, Biagini A, Sabini E, Valerio L, Giani C, Passannanti $P$ Pontillo-Contillo B, Battaglia V, Mazzeo S, et al. Lenvatinib and other tyrosine kinase inhibitors for the treatment of radioiodine refractory, advanced, and progressive thyroid cancer. Onco Targets Ther. 2016;9:6467-77.

17. Eichelberg C, Vervenne WL, De Santis M, Fischer von Weikersthal L, Goebell PJ, Lerchenmuller C, Zimmermann U, Bos MM, Freier W, Schirrmacher-Memmel S, et al. SWITCH: a randomised, sequential, open-label study to evaluate the efficacy and safety of Sorafenib-sunitinib versus Sunitinib-sorafenib in the treatment of metastatic renal cell Cancer. Eur Urol. 2015;68(5):837-47.

18. Busch J, Seidel C, Kempkensteffen C, Johannsen M, Wolff I, Hinz S, Magheli A, Miller K, Grunwald V, Weikert S. Sequence therapy in patients with metastatic renal cell carcinoma: comparison of common targeted treatment options following failure of receptor tyrosine kinase inhibitors. Eur Urol. 2011:60(6):1163-70.

19. Sugawara S, Oizumi S, Minato K, Harada T, Inoue A, Fujita Y, Maemondo M, Yoshizawa H, Ito K, Gemma A, et al. Randomized phase II study of concurrent versus sequential alternating gefitinib and chemotherapy in previously untreated non-small cell lung cancer with sensitive EGFR mutations: NEJ005/TCOG0902. Ann Oncol. 2015;26(5):888-94.

20. Lee YS, Kim SM, Kim BW, Chang HJ, Kim SY, Park CS, Park KC, Chang HS. Anti-cancer effects of HNHA and Lenvatinib by the suppression of EMT-mediated drug resistance in Cancer stem cells. Neoplasia. 2018; 20(2):197-206.

21. Park KC, Kim SM, Jeon JY, Kim BW, Kim HK, Chang HJ, Lee YS, Kim SY, Choi SH, Park CS, et al. Synergistic activity of N-hydroxy-7-(2-naphthylthio) Heptanomide and Sorafenib against Cancer stem cells, anaplastic thyroid Cancer. Neoplasia. 2017:19(3):145-53.

22. Ueno T, Endo K, Kondo S, Wakisaka N, Murono S, Ito M, Yoshizaki T. Factors affecting outcomes of alternating chemoradiotherapy for nasopharyngeal cancer. Ann Otol Rhinol Laryngol. 2014;123(7):509-16. 
23. Romaguera JE, Wang M, Feng L, Fayad LE, Hagemeister F, McLaughlin P, Rodriguez MA, Fanale M, Orlowski R, Kwak LW, et al. Phase 2 trial of bortezomib in combination with rituximab plus hyperfractionated cyclophosphamide, vincristine, doxorubicin, and dexamethasone alternating with bortezomib, rituximab, methotrexate, and cytarabine for untreated mantle cell lymphoma. Cancer. 2018;124(12):2561-9.

24. Montemayor-Garcia C, Hardin H, Guo Z, Larrain C, Buehler D, Asioli S, Chen $\mathrm{H}$, Lloyd RV. The role of epithelial mesenchymal transition markers in thyroid carcinoma progression. Endocr Pathol. 2013;24(4):206-12.

25. Lan L, Luo Y, Cui D, Shi BY, Deng W, Huo LL, Chen HL, Zhang GY, Deng LL. Epithelial-mesenchymal transition triggers cancer stem cell generation in human thyroid cancer cells. Int J Oncol. 2013;43(1):113-20.

26. Zhang P, Sun Y, Ma L. ZEB1: at the crossroads of epithelial-mesenchymal transition, metastasis and therapy resistance. Cell Cycle. 2015;14(4):481-7.

27. Meidhof S, Brabletz S, Lehmann W, Preca BT, Mock K, Ruh M, Schuler J, Berthold M, Weber A, Burk U, et al. ZEB1-associated drug resistance in cancer cells is reversed by the class I HDAC inhibitor mocetinostat. EMBO Mol Med. 2015;7(6):831-47.

28. Zhou G, Zhang F, Guo Y, Huang J, Xie Y, Yue S, Chen M, Jiang H, Li M. miR200c enhances sensitivity of drug-resistant non-small cell lung cancer to gefitinib by suppression of PI3K/Akt signaling pathway and inhibites cell migration via targeting ZEB1. Biomed Pharmacother. 2017;85:113-9.

29. Deng YR, Liu WB, Lian ZX, Li X, Hou X. Sorafenib inhibits macrophagemediated epithelial-mesenchymal transition in hepatocellular carcinoma. Oncotarget. 2016;7(25):38292-305.

30. Kurimoto R, Iwasawa S, Ebata T, Ishiwata T, Sekine I, Tada Y, Tatsumi K, Koide S, Iwama A, Takiguchi Y. Drug resistance originating from a TGF-beta/ FGF-2-driven epithelial-to-mesenchymal transition and its reversion in human lung adenocarcinoma cell lines harboring an EGFR mutation. Int J Oncol. 2016;48(5):1825-36.

31. Wilhelm SM, Carter C, Tang L, Wilkie D, McNabola A, Rong H, Chen C, Zhang X, Vincent P, McHugh M, et al. BAY 43-9006 exhibits broad spectrum oral antitumor activity and targets the RAF/MEK/ERK pathway and receptor tyrosine kinases involved in tumor progression and angiogenesis. Cancer Res. 2004;64(19):7099-109.

32. Glen H, Mason S, Patel H, Macleod K, Brunton VG. E7080, a multi-targeted tyrosine kinase inhibitor suppresses tumor cell migration and invasion. BMC Cancer. 2011;11:309.

33. Tohyama O, Matsui J, Kodama K, Hata-Sugi N, Kimura T, Okamoto K, Minoshima Y, Iwata M, Funahashi Y. Antitumor activity of lenvatinib (e7080): an angiogenesis inhibitor that targets multiple receptor tyrosine kinases in preclinical human thyroid cancer models. J Thyroid Res. 2014;2014:638747.

34. Zhu YJ, Zheng B, Wang HY, Chen L. New knowledge of the mechanisms of sorafenib resistance in liver cancer. Acta Pharmacol Sin. 2017;38(5):614-22.

35. Adjibade P, St-Sauveur VG, Quevillon Huberdeau M, Fournier MJ, Savard A, Coudert L, Khandjian EW, Mazroui R. Sorafenib, a multikinase inhibitor, induces formation of stress granules in hepatocarcinoma cells. Oncotarget. 2015;6(41):43927-43

36. Qi L, Song W, Li L, Cao L, Yu Y, Song C, Wang Y, Zhang F, Li Y, Zhang B, et al. FGF4 induces epithelial-mesenchymal transition by inducing storeoperated calcium entry in lung adenocarcinoma. Oncotarget. 2016;7(45): 74015-30.

37. Ryu SH, Heo SH, Park EY, Choi KC, Ryu JW, Lee SH, Lee SW. Selumetinib inhibits melanoma metastasis to mouse liver via suppression of EMT-targeted genes. Anticancer Res. 2017;37(2):607-14.

\section{Ready to submit your research? Choose BMC and benefit from:}

- fast, convenient online submission

- thorough peer review by experienced researchers in your field

- rapid publication on acceptance

- support for research data, including large and complex data types

- gold Open Access which fosters wider collaboration and increased citations

- maximum visibility for your research: over $100 \mathrm{M}$ website views per year

At BMC, research is always in progress.

Learn more biomedcentral.com/submissions 